\title{
Modeling and Simulation of Laminar Jet Diffusion Flames of Methyl Butanoate
}

\author{
Silvia Barcelos Machado ${ }^{1}$ \\ Graduate Program in Applied Mathematics, UFRGS, Porto Alegre, RS \\ Adelaida Otazu Conza ${ }^{2}$ \\ Graduate Program in Applied Mathematics, UFRGS, Porto Alegre, RS \\ Álvaro Luiz de Bortoli ${ }^{3}$ \\ Graduate Program in Applied Mathematics, UFRGS, Porto Alegre, RS \\ Graduate Program in Chemical Engineering, UFRGS, Porto Alegre, RS
}

\begin{abstract}
The aim of this work is to obtain the mass fraction of the species involved in a two step mechanism for a biodiesel surrogate, methyl butanoate (MB). For this, the solution for the one-step and two-step models was used and the flow equations were solved. The equations were discretized by the finite difference method and were integrated by the Simplified Runge-Kutta method. The results obtained agree with data from the literature.
\end{abstract}

Keywords. Methyl butanoate, Jet diffusion flame, Burke-Schumann solution, CFD, Simplified Runge-Kutta method.

\section{Introduction}

Biofuels are solid, liquid or gaseous fuels that are produced from starch, sugar, oil and raw materials obtained through the cultivation of wheat, corn, sugar cane, among others [13]. Biofuels are produced from biomass and waste materials from the food industry, forestry, etc. They include: bioethanol derived from lignocellulose, biodiesel obtained by refining used vegetable oils, etc.

Biodiesel can be reasonably represented by simpler surrogate fuels that are of the same ester class [3,7]. For example, $\mathrm{MB}\left(\mathrm{C}_{5} \mathrm{H}_{10} \mathrm{O}_{2}\right)$ contains the essential chemical structure of large chain fatty acids, and is a reasonable surrogate for flames of biodiesel at high temperatures.

Models of reactive flowns using Computational Fluid Dynamics (CFD) equations are complex [10]. The mathematical formulation of CFD consists of coupled nonlinear partial differential equations such as: continuity, momentum and mixture fraction, which can be discretized using, e.g., methods as finite differences and finite elements $[6,8]$.

\footnotetext{
${ }^{1}$ sbmmtm@gmail.com

22o.conzaadelaida@gmail.com

${ }^{3}$ dbortoli@mat.ufrgs.br
} 
The aim of this work is to model the mass fraction of the biofuel surrogate, $\mathrm{MB}$, for one and two-step mechanism and solve the flow equations. The solution of these equations is approximated by the Simplified Runge-Kutta method.

\section{Model formulation}

\subsection{Burke-Schumann solution}

Diffusion flames can be described by the conservative scalar $Z$, which represents the mixture fraction. The mass fraction of the unburned fuel is $Y_{F, u}=Y_{F, 1} Z$, where $Y_{F, 1}$ is the mass fraction of the fuel in the initial stream. Since $(1-Z)$ represents the mass fraction of the oxidant, we can also write $Y_{O 2, u}=Y_{O 2,2}(1-Z)$, where $Y_{O 2, u}$ is the mass fraction of the unburnt oxidant and $Y_{O 2,2}$ is its mass fraction.

Considering the global one-step reaction $[11,12]$

$$
\nu_{F}[F]+\nu_{O_{2}}\left[O_{2}\right] \rightleftharpoons \nu_{C O_{2}}\left[C O_{2}\right]+\nu_{H_{2} O}\left[H_{2} O\right],
$$

where $\nu_{i}$ is the stoichiometric coefficient of the species $i$, we have that the relation between the mass fractions of the fuel and the oxidant is given by $\frac{d Y_{i_{1}}}{\nu_{i_{1}} W_{i_{1}}}=\frac{d Y_{i_{2}}}{\nu_{i_{2}} W_{i_{2}}}$, where $W_{i}$ represents the molecular weight of the species $i$.

Integrating the last equation, with the subscripts $i_{1}=F$ and $i_{2}=O_{2}$, between the initial unburnt state and another posterior state, we have $\frac{Y_{F}-Y_{F, u}}{\nu_{F} W_{F}}=\frac{Y_{O_{2}}-Y_{O_{2}, u}}{\nu_{O_{2}} W_{O_{2}}}$. Then

$$
\nu Y_{F}-Y_{O_{2}}=\nu Y_{F, u}-Y_{O_{2}, u},
$$

where $\nu=\frac{\nu_{\mathrm{O}_{2}} W_{\mathrm{O}_{2}}}{\nu_{F} W_{F}}$ is the stoichiometric ratio.

From equation (2), we write the mixture fraction $Z=\frac{\nu Y_{F}-Y_{O_{2}}+Y_{O_{2}, 2}}{\nu Y_{F, 1}+Y_{O_{2}, 2}}$. For a stoichiometric mixture, $\nu Y_{F}=Y_{O_{2}}$. Then the stoichiometric mixture fraction is given by $Z_{s t}=\left(1-\nu \frac{Y_{F, 1}}{Y_{O_{2}, 2}}\right)^{-1}$.

The solution of the global one-step mechanism can be approximated by the BurkeSchumann solution, which describes the mass fraction of the species involved in the reaction with respect to the mixture fraction $\mathrm{Z}$ [2]. For $Z \leq Z_{s t}$, combustion ends when all fuel is consumed and the misture fraction result in:

$$
\left\{\begin{array}{l}
Y_{F, b}=0 ; \\
Y_{O_{2}, b}=Y_{O_{2}, u}\left(1-\frac{Z}{Z_{s t}}\right) ; \\
Y_{C O_{2}, b}=Y_{C O_{2}, s t} \frac{Z}{Z_{s t}} \\
Y_{H_{2} O, b}=Y_{H_{2} O, s t} \frac{Z}{Z_{s t}}
\end{array}\right.
$$

For $Z>Z_{s t}$, the combustion is complete when all oxygen is consumed and the equations result in: 


$$
\left\{\begin{array}{l}
Y_{F, b}=Y_{F, 1}\left(\frac{Z-Z_{s t}}{1-Z_{s t}}\right) \\
Y_{O_{2}, b}=0 \\
Y_{C O_{2}, b}=Y_{C O_{2}, s t}\left(\frac{1-Z}{1-Z_{s t}}\right) \\
Y_{H_{2} O, b}=Y_{H_{2} O, s t}\left(\frac{1-Z}{1-Z_{s t}}\right)
\end{array}\right.
$$

where $Y_{C O_{2}, s t}=\frac{\nu_{C O_{2}} W_{C O_{2}}}{\nu_{F} W_{F}} Y_{F, 1} Z_{s t}$ and $Y_{\mathrm{H}_{2} \mathrm{O}, s t}=\frac{\nu_{\mathrm{H}_{2} \mathrm{O}} W_{\mathrm{H}_{2} \mathrm{O}}}{\nu_{F} W_{F}} Y_{F, 1} Z_{s t}$.

In a mechanism of more than one step, we write the mixture fraction in the form: $Z=Z_{1}+Z_{2}+\cdots+Z_{n}$, where $n$ are the components. In terms of the mass fraction of the species [1], we have:

$$
Z_{1}=\frac{Y_{F}}{Y_{F, 1}} ; Z_{k}=\frac{\nu_{F} W_{F}}{\nu_{k} W_{k}} \frac{Y_{k}}{Y_{F, 1}} ; k=2, \cdots, n
$$

so that when we replace (5) in the mass fraction equation of the species

$$
\rho \frac{\partial Y_{k}}{\partial t}+\rho \vec{u} \cdot \vec{\nabla} Y_{k}=\vec{\nabla} \cdot\left(\rho D \vec{\nabla} Y_{k}\right) \pm \dot{\omega}_{k} ; k=1, \cdots, n
$$

we obtain

$$
\rho \frac{\partial Z_{k}}{\partial t}+\rho \vec{u} \cdot \vec{\nabla} Z_{k}=\vec{\nabla} \cdot\left(\rho D \vec{\nabla} Z_{k}\right) \pm \dot{\omega}_{k} ; k=1, \cdots, n .
$$

Adding the $n$ equations in (7) gives the equation of the mixture fraction described in (8). The solution of the set of $n$ equations is obtained numerically.

\subsection{Flow equations}

In order to formulate the system of equations that model the flow of a free jet, we consider the following hypotheses: i) the flow is two-dimensional and laminar; ii) the flow regime is permanent and the fluid is incompressible; iii) field forces are negligible.

The flow will be described by the dimensionless equations of continuity, momentum, pressure and mixture fraction [5]:

$$
\left\{\begin{array}{l}
\nabla \cdot \vec{u}=0 \\
\frac{\partial \vec{u}}{\partial t}+\vec{u} \cdot \vec{\nabla} \vec{u}=-\frac{1}{\rho} \vec{\nabla} p+\frac{1}{R e} \nabla^{2} \vec{u} \\
\nabla^{2} p=2\left(\frac{\partial u}{\partial x} \frac{\partial v}{\partial y}-\frac{\partial u}{\partial y} \frac{\partial v}{\partial x}\right) \\
\frac{\partial Z}{\partial t}+\vec{u} \cdot \vec{\nabla} Z=\frac{1}{R e S c} \nabla^{2} Z
\end{array}\right.
$$

where $R e=\frac{u L}{\nu}$ is the Reynolds number, which determines the fluid flow regime, $S c=\frac{\nu}{D}$ is the Schmidt number, which relates the viscosity and the mass diffusivity of the fluid. 
Considering the domain $\Omega=\{(x, y): 0 \leq x \leq 1,0 \leq y \leq 0.1\}$, the initial and boundary conditions are given by:

$$
\left\{\begin{array}{l}
\vec{u}=(u, v)=(1,0), p=Z=1, \text { for } t=0, \forall \Omega ; \\
u=Z=\left\{\begin{array}{l}
1, \text { if } 0.045 \leq x \leq 0.055 \\
0, \text { otherwise }
\end{array}, v=\frac{\partial p}{\partial x}=0, \text { for }(0, y) \in \Omega ;\right. \\
u=v=Z=\frac{\partial p}{\partial x}=0, \text { for }(x, 0) \in \Omega ; \\
u=v=Z=\frac{\partial p}{\partial y}=0, \text { for }(x, 0.1) \in \Omega ; \\
\frac{\partial u}{\partial x}=\frac{\partial v}{\partial x}=\frac{\partial Z}{\partial x}=0, p=1, \text { for }(1, y) \in \Omega .
\end{array}\right.
$$

\subsection{Numerical formulation}

The Simplified Runge-Kutta method [1] is used because of the small number of operations required. The coefficients of the method were chosen to obtain a solution with high temporal precision. For the system of differential equations $\frac{\partial \vec{W}}{\partial t}=-\vec{R}$, the Simplified Runge-Kutta method is:

$$
\left\{\begin{array}{l}
\vec{W}_{(i, j)}^{(0)}=\vec{W}_{(i, j)}^{(n)} \\
\vec{W}_{(i, j)}^{(k)}=\vec{W}_{(i, j)}^{(0)}-\alpha_{r} \Delta t \vec{R}_{(i, j)}^{(k-1)} \\
\vec{W}_{(i, j)}^{(n+1)}=\vec{W}_{(i, j)}^{(k)} ;
\end{array}\right.
$$

where $\vec{R}$ is the vector representing the system of equations evaluated at points $(i, j)$ of the domain, in stage $k, k=1, \ldots, n ; \vec{W}$ contains the variables of interest of the problem; $\Delta t$ is the time step and $\alpha_{r}$ are the coefficients of the method. For second order temporal approximation with 3 stages, we take $\alpha_{1}=\alpha_{2}=0.5$ and $\alpha_{3}=1$.

\section{$3 \quad$ Numerical results}

The equations of the flow were discretized using the finite difference method, in which one replaces the derivates of the differential equations by approximations involving only numerical values $[4,14]$. Consider a jet as shown in Figure 1 , in the domain $\Omega$, whose nozzle diameter is $d \simeq 0.01$. A non-uniform mesh was used, refined on $\Omega$ at the beginning and centerline of the jet, with $151 \times 51$ points. The dimensionless numbers taken were $R e=2400$ and $S c=0.5$. The Simplified Runge-Kutta method was applied using $\Delta t=10^{-6}$, from which numerical data for the laminar diffusion flame mixture fraction were obtained.

Figure 1 shows the isolines of mixture fraction for a laminar jet diffusion flame of MB.

A numerical result of mass fractions for MB diffusion flame can be obtained from the global two-step mechanism:

$$
\begin{aligned}
{\left[\mathrm{C}_{5} \mathrm{H}_{10} \mathrm{O}_{2}\right]+4\left[\mathrm{O}_{2}\right] } & \rightleftharpoons 5[\mathrm{CO}]+5\left[\mathrm{H}_{2} \mathrm{O}\right] ; \\
{[\mathrm{CO}]+\frac{1}{2}\left[\mathrm{O}_{2}\right] } & \rightleftharpoons 5\left[\mathrm{CO}_{2}\right] .
\end{aligned}
$$

Figure 2 shows the mass fraction as a function of the mixture fraction of the species of the laminar jet diffusion flame in the centerline of the jet. The maximum values for the 


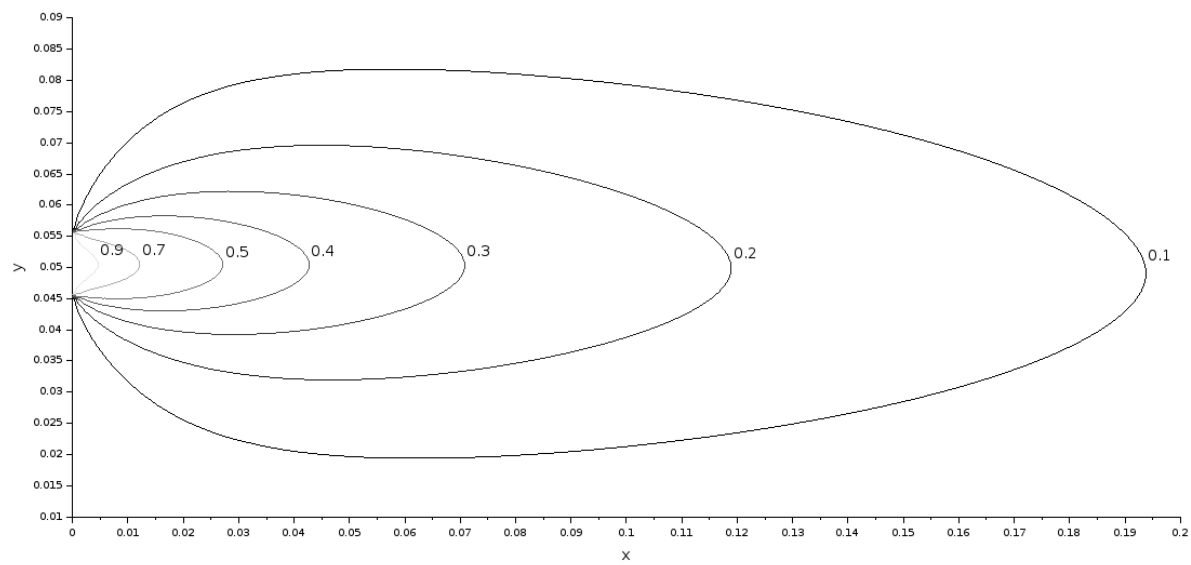

Figure 1: Isolines of the mixture fraction for diffusion flame of MB.

$\mathrm{H}_{2} \mathrm{O}, \mathrm{CO}_{2}$ and $\mathrm{CO}$ species in the stoichiometric mixture fraction $\left(Z_{s t} \cong 0.1025\right)$ are the ideal conditions for burning.

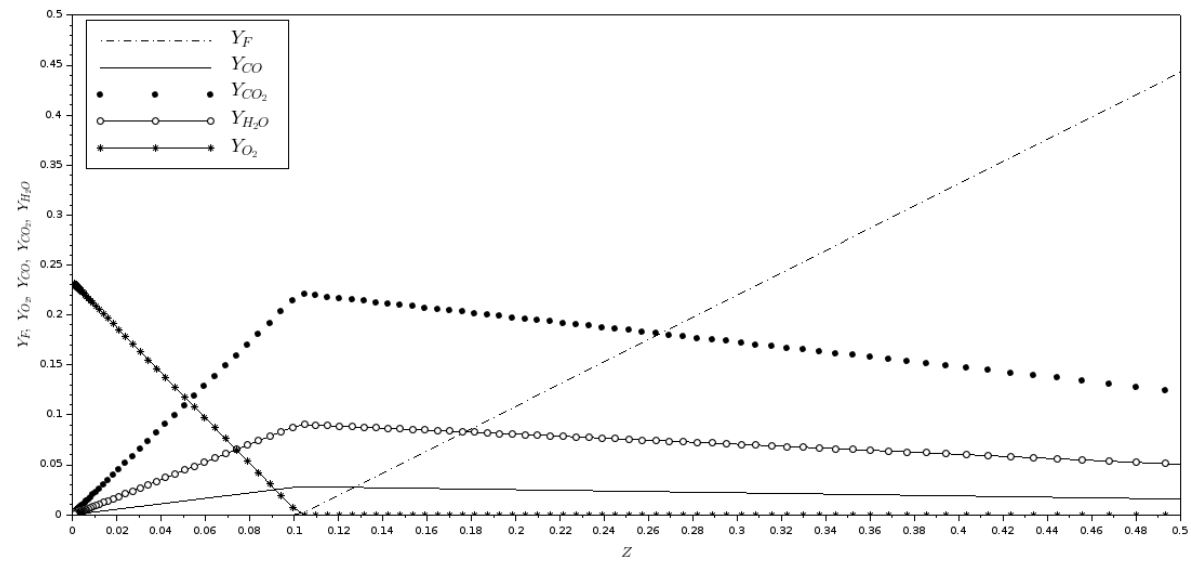

Figure 2: Mass fraction of the species along the centerline of the jet along $\mathrm{Z}$.

The truncation error of the mixture fraction equation, for infinity norm is given by:

$$
e_{\infty}^{N}=\max _{i=1, \ldots, N_{p}}\left|e_{i}^{N}\right|,
$$

where $e_{i}^{N}$ is the mixture fraction difference in the iteration $N$ and $N-1$, for $\mathrm{N}=2,3, .$. , and $N_{p}$ is the number of points of the mesh. The results shown in Table 1 indicate the 
increase in accuracy of the Simplified Runge-Kutta method for the mixture fraction in the infinite norm.

Table 1: Error for mixture fraction equation.

\begin{tabular}{cc}
\hline Iteration Error & Error $\left(E_{\infty}\right)$ \\
\hline 500000 & $1.8139601358664603 \mathrm{E}-007$ \\
1000000 & $3.5497390957206498 \mathrm{E}-008$ \\
1500000 & $2.8048385565526690 \mathrm{E}-008$ \\
2000000 & $1.6826276684478447 \mathrm{E}-008$ \\
2500000 & $9.1952749303736425 \mathrm{E}-009$ \\
3000000 & $4.8239832250301928 \mathrm{E}-009$ \\
4000000 & $1.2876340206753600 \mathrm{E}-009$ \\
5000000 & $3.6559603998689849 \mathrm{E}-010$ \\
6000000 & $1.1934498354848611 \mathrm{E}-010$ \\
\hline
\end{tabular}

\section{Conclusion}

In this work, we model a jet diffusion flame of methyl butanoate using a two-step mechanism to determine the mass fraction of the species with respect to the mixture fraction, given through the flow equations. The Burke-Schumann solution is used for the solution of the one step model. The numerical solution of the two step model and flow equations was obtained using the Simplified Runge-Kutta method and the convergence is shown in Table 1.

According to $[9,15]$, the results obtained for the mass fractions of $\mathrm{MB}, \mathrm{O}_{2}, \mathrm{CO}, \mathrm{CO}_{2}$ and $\mathrm{H}_{2} \mathrm{O}$ are coherent.

\section{Acknowledgment}

This research is being developed at UFRGS. The first and second authors gratefully acknowlege the financial support from CAPES, Coordenação de Aperfeiçoamento de Pessoal de Nível Superior, while Prof. De Bortoli from CNPq, Conselho Nacional de Desenvolvimento Científico e Tecnológico under grant 306768/2018-6.

\section{References}

[1] A. L. de Bortoli, G. S. L. Andreis, F. N. Pereira. Modeling and simulation of reactive flows. Elsevier Science Publishing, USA, 2015.

[2] S. P. Burke, T. E. W. Schumann. Diffusion flames. Industrial Engineering Chemistry, 20:998-1004, 1948. DOI: 10.1016/s1062-2888(65)80003-x. 
[3] S. Dooley, H. J. Curran, J.M. Simmie. Autoignition measurements and a validated kinetic model for the biodiesel surrogate, methyl butanoate. Combustion and Flame, 153:2-32, 2008. DOI:10.1016/j.combustflame.2008.01.005.

[4] J. H. Ferziger, M. Peric. Computacional methods for fluid dynamics. 3ed. Springer, 2002.

[5] W. F. Hughes, J. A. Brighton. Fluid dynamics. 3 ed. Schaum's Outline, McGraw-Hill Education. 1999.

[6] M. G. Larson, F. Bengson. The Finite Element Method: Theory, Implementation, and Applications. Springer, 2013.

[7] W. K. Metcalfe, S. Dooley, H. J. Curran, J. M. Simmie, A. M. El-Nahas, M. V. Navarro. Experimental and Modeling Study of $\mathrm{C}_{5} \mathrm{H}_{10} \mathrm{O}_{2}$ Ethyl and Methyl Esters. Journal of Physical Chemistry A, 111:4001-4014, 2007. DOI: 10.1021/jp067582c.

[8] K. W. Morton, D. F. Mayers. Numerical Solution of Partial Differential Equations. Cambridge University Press, 2005.

[9] U. Niemann, R. Seiser, K. Seshadri. Ignition and extinction of low molecular weight esters in nonpremixed flows. Combustion Theory and Modeling, 14:875-891, 2010. DOI: $10.1080 / 13647830.2010 .517275$.

[10] E. S. Oran, J. P. Boris. Numerical Simulation of Reactive Flow, 2 ed. Cambridge University Press, 2001.

[11] N. Peters. Fifteen Lectures on Laminar and Turburlent Combustion. Germany: Ercoftac Summer School, 1992. Accessed on March 10, 2019. <https://www.itv.rwthaachen.de/fileadmin/Downloads/Summerschools/SummerSchool.pdf $>$.

[12] N. Peters. Turbulent Combustion. Cambridge University Press, 2006.

[13] S. Royal. Ustainable biofuels: prospects and challenges. 2008.

[14] J. C. Strikwerda. Finite difference schemes and partial differential equations. Society for Industrial and Applied Mathematics. Philadelphia, 1974.

[15] W. R. Zeng, W. K. Chow. A note on modeling combustion of common fuels with mixture fraction. International Journal on Engeneering Performance-Based Fire Codes, 10:6-11, 2011. 